\title{
Choice and Meaning in the Quantum Universe.
}

\author{
Henry P. Stapp \\ Theoretical Physics Group \\ Physics Division \\ Lawrence Berkeley Laboratory \\ 1 Cyclotron Road \\ Berkeley, California 94720
}

Invited presentation. Congrès: Science et 'Tradition; Perspectives Transdisciplinaires, Ouvertures vers le XXI ème siècle. à 1' U.N.E.S.C.O. du 2 au 6 Décembre 1991.

"This work was supported by the Director, Office of Energy Research, Office of High Energy and Nuclear Phy'sics, Division of High Energy Physics of the U.S. Department of Energy under Contract DE-AC03-765F00098. 


\section{Choice}

How does the world come to be just what it is, and not something else? Classical physics offers only a partial answer. It says that the deterministic laws of nature fix everything over all of spacetime in terms of things at a single instant of time. But the remaining question is then: What fixes things at this single instant of time? What determines the initial conditions?

Classical physics provides no answer at all to this question, or only the equivalent answer 'God', where God is the narne of whatever it is that fixes those things that are not fixed by the laws of nature, as they are currently understood by scientists. I shall call by the name 'choice' any fixing of something that is left free by the laws of nature, as they are currently understood.

Classical physics is not the ultimate scientific theory. It fails at the level of atomic phenomena, and has been replaced by quantum theory. However, the quantum laws, unlike the classical laws, are indeterministic: they fix not what actually happens, but only the probabilities for the various things that might happen. That is, quantum theory, in its orthodox form, provides no answer to the further question: What fixes what actually does happen?

Physicists have proposed four fundamentally different answers to this question. In the first part of this talk I shall describe these four possibilities. However, one thing is immediately clear. If, at the deepest level, the laws of nature are isasically indeterministic, like the laws of quantum theory, then, by definition, choices are not confined to the beginning of time: they must occur under more general conditions. In this case a central question in man's search for an understanding of nature, and his place within it, must be this: Under what conditions are choices made, and what role, if any, do human beings play in the generation of choice?

\section{Bohr's Approach}

The first of the four proposals concerning choice is agnostic: it declines to address the issue of where choices occur, on the grounds that this question does not lie within the province of science, or at least within the province of physics. This is the approach of Niels Bolnr, whose general orientation is characterized by the following quotations: 
"The task of science is both to extend the range of our experience and reduce it to order ..." . "In physics ... our problern consists in the coordination of oux experience of the external world ..." 2 . "In our description of naturc the purpose is not to disclose the real essence of phenomena but only to track down as far as possible relations between the multifold aspects of our experience" ${ }^{3}$. As regards the quanturn formalism itself Bohr says:

"Thr sole aim [of the quantum formalism] is the comprehension of observations obtuined under experimental conditions described by simple physical concepts" "Strictly speaking, the mathematical formalism of quantum mechanics and electrodynariucs merely offers rules of calculation for the deduction of expectations about observations obtained under well-defined experimental conditions specified by classical physical concepts" ${ }^{\text {5 }}$.

The quantum formaiism referred to by Bohr works in the following way ${ }^{6}$ Let $A$ represent a description in terms of classical concepts of the preparation of an atomic system - - i.e., a description in terms of the concepts of classical physics of the construction and placement of the preparing devices. Let $B$ represent a description in terms of classical concepts of a possible response of the detection system - - i.e., a set of specifications that will allow technically trained observers to determine whether an observed response lies in the specified class $B$. Then the basic assumption of quantum thoory is that, under appropriate conditions, there are mappings

$$
A \rightarrow\left|\psi_{A}><\psi_{A}\right| \equiv \rho_{A}
$$

and

$$
B \rightarrow\left|\psi_{B}><\psi_{B}\right|=\delta_{E},
$$

from classical descriptions to operators in a Hi'bert space, such that the probability that a result meeting specifications $B$ will scur under the conditions $A$ is given by the formula

$$
\begin{aligned}
P(A: B) & =\mid\left\langle\psi_{B}\left|\psi_{A}>\right|^{2}\right. \\
& =\left\langle\psi_{B}\right| \psi_{A}>\left\langle\psi_{A} \mid \psi_{B}\right\rangle \\
& =\operatorname{Tr}_{A} \delta_{B} .
\end{aligned}
$$

In accordance with Bohr's precepts, this formalism is nothing but a set of rules 
for computing expectations pertaining to observations obtained under conditions specified in terms of classical concepts.

Bohr claimed that predictions computed essentially in this way provide all of the confirmable predictions about atomic phenomena that are possible in principle, and that quantum theory provides, therefore, a complete description of atomic phenomena: no theory based on some purported 'more detailed' description of the atoms can ever, according to Bohr, yield additional confirmable predictions about phenomena of this kind.

\section{Everett's Approach}

Bohr clas.ned that the description of atomic (and perhaps subatomic) systems in terms of quantum states is complete. Since the physical universe is composed, in some sense, of atomic (and subatomic) particles it seerns reasonable to try to represent the entire universe in the same way that one represents a collection of atoms, namely by an operator in a Hilbert space. However, in doing so it is important to recognize that most of the degrees of freedom referred to by such an operator represent properties that are extremely ephemeral: they are properties that are not directly observable by human beings, and are extremely fleeting on the time scale of human experience. The full universe consists therefore of an exceedingly thin veneer of relatively sluggish, directly observable properties resting on a vast ocean of rapidly fluctuating unobservable ones.

If one examines, theoretically, the evolution of the universe under the assumption that nature's process is governed exclusively by a Schroedinger equation, which is the norinal quantum law of evolution, then the following picture emerges: due to the local character of interaction, between particles the properties of nature in the thin veneer of local observable properties is continually splitting into a statistical mixture of classical wor!ds of the kind we observe. By a 'statistical mixture' I mean a collection of possibilities each having a definite statistical weight, where this statistical weight can be interpreted as the probaw bility that this particular possibility will be the one that is actually realized in nature.

The proposal of Heisenberg and Dirac, which will be described later, asserts 
that nature singles out and actualizes one observable branch from among the emerging set of possible ones. Everett's counter-proposal is that no such choice is ever made, but that rather the character of human consciousness is such that each individual realm of human experience can accomodate only a single one of these branches, even though all the branches exist together in the fullness of nature. Thus in Everett's picture of nature conly one choice need ever be made, namely the choice of the initial state of the universe. This initial statercould be taken to be some featureless state, on the grcunds of a lack of sufficient reason for any' specific feature. 'Then the particularness of the perceived universe observed by any individual person would not be a reflection of any corresponding particularness of the initial state of the universe: it would not be, as in classical physics, merely a transformed expression of the particularness present already at earlier times. Rather the observed particularness would be the particularness of one individual branch of the universe. This branch is generated out of a 'quantum soup" by the deterministic laws of quantum evolution, with no intervention of choice.

Everatt's proposal ${ }^{7}$ has, for physicists, the attraction that it makes quantum theory complete in principle. The theory would, if valid, cover, in principle, not only atomic phenomena but also biological and cosmological processes, for example. However, even the proponents of Everett's theory emphasize that the technical details of this interpretation need to be spelled out in more detail. The problem, basically, is the clash between the continuous character of the description of nature provided by the quantum state and the discrete character of human experience. The Everett universe at the observable level probably does not separate into well-defined discrete branches. The various 'branches' appear to blend continuously into each other, due to the basically continuous character of the elernentary scattering and decay processes. In the standard applications of the quantum formalism to atomic phenomena a human agent plays a crucial role of setting up specifications for identifying particular classess of physical events. But in Everett's quantum world the human observers and their devices tend to become amorphous distributions of properties. Consequently, no sharp separation of the observable aspects of nature into discrete well-defined branches has yet been demonstrated. This leaves the technical viability of Everett's proposal open to serious doubt. 
This problem of the reconciliation of the discreteness of the perceived world with the amorphous character of its purely quantur. description is cleanly resolved by the proposal of David Bohm. 


\section{Bohm's Pilot-Wave Proposal}

The quantum formalism is fundamentally statistical in character. Hence it is reasonable to postulate the existence in nature of the actual things that the quartum probabilities are probabilities of. These things will then specify what actually occurs.

David Bohm ${ }^{8}$ has constructed a model of this kind. In his model there is an ordinary classical world of the kind described in classical physics, and, in addition, also a quaxitum state. This state is supposed to exist as a physically real thing, not merely as an idea in the minds of scientists. It specifies an extra force that acts on each of the particles of the classical world, and causes them to behave in a way compatible with the statistical predictions of quanturn theory.

Bohm's model is simple and instructive. It shows that we need not cling to the idea, advanced by the founders of quantum theory, that nature cannot be described in a thoroughly comprehensible way in terms of properties that are always well defined and that evolve in accordance with well-defined deterministic laws.

Bohm's model does violate one of the basic precepts of classical physics: the force on a particle located at a point generally depends strongly upon the precise locations, at that very instant, of many other particles in the universe. This instantaneous connection contradicts the idea of classical relativistic physics that no influence can act over a spacelike interval - - i.e., faster than light. On the other hand, a now-famous theorem due to John Bell ${ }^{9}$ shows that no deterministic theory of this general kind can exclude faster-than-light influences, if it is to reproduce the predictions of quantum theory. Bell's result can be extended also to indeterministic theories. ${ }^{10}$ Thus this nonlocal feature ought not be regarded as objectionable, provide all the observable properties conform to relativistic principles, as they indeed do in Bohm's relativistic model.

Bohm's model does however retain one feature of classical physics that can be regarded as objectionable, at least aesthetically. This is the need for an arbitrary-looking choice of initial conditions. In particular, some definite initial position for each of the particles in the universe must be chosen. The idea of such an immensely detailed choice suddenly emerging out of nothing at all seems utterly unreasonable. In the alternative proposal of Heisenberg and Dirac, to be 
described next, the choices are distributed over space and time, and each choice is made within a specific physical context.

\section{The Heisenberg-Dirac Proposal}

The picture of nature most nearly in line with quantum theory as it is used in practice is that of Heisenberg and Dirac. Heisenberg says: "The observation itself changes the probability function discontinuously; it selects of all possible events the actual one that has taken place. ... the transition from the "possible" to the "actual" takes place during the act of observation. If we want to describe what happens in an atomic event, we have to realize that the word "happens" can apply only to the observation, not to the state of affairs between two observations. It applies to the physical, not the psychic act of observation, and we may say that the transition from the "possible" to the "actual" takes place as soon as the interaction of the object with the measuring device, and thereby with the rest of the world, has come into play, it is not connected with the act of registration of the result in the mind of the observer." 11

Heisenberg distinguishes what is actually happening in the physical world from representations of the physical situation in the minds of scientists. Strictly speaking, the quantum formalism pertains exclusively to the latter. However, the extreme precision of the predictions of quantum theory justifies our trying to think of nature herself as represented by a quantum state, which, however, must undergo a sudden 'quantum jump' in connection with each selection of an actual result from among the ones previously possible.

Dirac espouses a similar idea when he speaks of a 'choice' on the part of nature.

The intervention of 'choice' in the proposal of Heisenberg/Dirac is completely different from this intervention in the proposals of Everett and of Bohm. In Everett's model there need be no choice at all, except perhaps a choice of a featureless initial condition: all of the particularness that we observe in rature can be supposed to exist in a single branch that is generated in a completely deterministic way by deterministic laws of motion, but then mistakenly perceived to be the whole of nature by virtue of a limitation in the capacity of each individual human consciousness. In Bohm's model, on the other hand, all choice 
is confined to a single stupendous choice that can be conceived to be made at 'the beginning of time', or at some time in the far distant past. In the Heisenberg/Dirac proposal the choice of initial conditions can be, as in the Everett model, the choice of a featureless state. Then, over the course of time, choices are made that inject into the universe the particularness that we observe. Each choice in the present era is taken to he a choice from among the observable possible branches that are generated by the deterministic laws of quantum evolution. Under the condition that prior choices have been made, this process can be conceived to generate, at the level of local observable properties, a statistical mixture of reasonably distinct branches, some one of which will be selected.

The brain of an alert human observer is similar in an important way to a quantum detecting device: it can amplify small signals to large macroscopic effects. The Heisenberg/Dirac proposal, if taken seriously, must therefore be expected to entail quantum events in the brain that are analogous to the events that are postulated to occur in quantum detecting devices. On the other hand, a quantum event in the brain, if it occurs at the level of the entire brain, or a large part of it, could be incomparably more complex than the actualized state of a simple quantum detection device, simply because of the immensely greater complexity of the brain itself, as contrasted to a quantum measuring device.

Suppose the actualized state of the brain is really actualized. What can this mean? One possibility is that some characteristic feature of this state becomes an actual "experience". Such a physical feature, if correctly identified, could become the basis of the correspondence between the physical world described by the physicist and neurophysiologist, and the psychic world described by the psychologist. I shall return to this question after a consideration of the nature of meaning.

\section{Meaning}

The idea of meaning entails a sense of direction: a sense of endurance with refinement; a notion of a process that sustains and refines itself. Thus meaning demands mechanism: it demands a machinery that allows a form to be recreated in refined form. Endurance and reproducibility are essential: the form must endure long enough to activate and guide the machinery that sustains and refines it. 
States characterized by local observable properties have the required characteristics of endurance and reproducibility, whereas superpositions of such states do not: the interaction of these latter states with their environments quickly destroys the phase connections that define them, and they are consequently unable to reproduce themselves. Thus local observable properties, or properties similar to them, are the natural, and perhaps exclusive, carriers of meaning within the quantum universe.

From this point of view the quantum universe tends to create meaning: the quantum law of evolution continuously creates a vast ensemble of forms that can act as carriers of meaning; it generates a profusion of forms that have the capacity to sustain and refine themselves.

There are among the full set of quantum states that conceivably could be actualized a plethora of possibilities. Yet if we accept the ideas of Heisenberg and Dirac, or the direct evidence of our senses, the forms that actually are chosen are forms of an exceedingly special kind: they are forms that sustain themselves: the pointer on the measuring instrument swings to the right, and this form endures, not in an absolutely static state, but in a state that sustains an enduring semblance of itself.

This essential characteristic of the quantum event is shared by the only things we really know to be actual, our own experiences: each human experience is a form that actualizes itself as an enduring structure.

In a certain sense this property of the actualized forms is logically required. Consider a thousand dots arranged in a small square. Each of the conceivable possible arrangements constitutes a definite form. However, each of these forms is, at the purely intrinsic level, equivalent to every other one: there is no intrinsic distinction between them. Each one is different, but they are all intrinsically equivalent. To specify some significant difference one must go beyond the immediate intrinsic form itself.

Scientists, in their search for simplicity, endeavor to consider the physical universe as self-contained; as not requiring the intervention some outside agent. To achieve such an end any distinction made by nature between conceivable possible forms must be based on properties intrinsic to the quantum universe itself. One way to draw such a distinction is to consider each form on the basis 
of what it does, or produces in the quantum universe, rather than on the basis of what it is.

If this strategy is adopter then there is one logical distinction between forms that stands out from all others, in the sense that it does not appeal to any structure that lies outside the form itself. This is the property of a form to sustain itself.

From this point of view the proposal of Heisenberg and Dirac can be characterized in this way: the quantum choices are meaningful choices, where 'meaningful' is defined intrinsically, within the quantum system itself, without reference to any external criterion of meaning, in terms of sustainability. Each quantum choice pulls itself out of the quantum soup 'by its bootstraps'; it justifies itself by the meaning inherent in the sustainability of the form that is actualized. The 'meaning' of this choice is, then, not based upon anything lying outside the chosen form: it resides in the sustainability of that form itself.

This introduction of a notion of intrinsic meaning at the level of the elementary quantum event provides the rudiment of a general quantum conception of meaning based on the intrinsic criterion of sustainability.

Within the quantum formalism each Heisenberg/Dirac quantum choice is a grasping, as a unified whole, of a certain combination of possibilities that hang together as a local enduring form. 's 'he actualization of this form utilizes, and restructures, some of the quantum potentialities, and produces an immediate rearrangement of the possibilities available for the next event. The specific form of this rearrangement is fixed by the mathematics of quanturn theory.

A principal feature of this rearrangement of possibilities is that a choice made in one region instantly affects the possibilities available in far away regions. If the potentiality for a particle to be detected in one detector is actualized, then the potentiality for this particle to be detected in a far-away region immediately vanishes. Thus the quantum choice is, on the one hand, a local a aur, because it actualizes a particular meaningful form in a local region of spacetime. On the other hand, the bookkeeping system is global: an adjustment of possibilities is immediately made over the entire spacetime manifold. Thus the basic process of choice is fundamentally global, but it creates locally defined meaning. 


\section{Ramifications}

The foregoing discussion of meaning offers something that science is expected ultimately to provide, and that is desperately needed today, namely the basis of a 'Weltanschauung', or world view, that is fully compatible with the available scientific evidence, and which counters the corrosive mechanical world view that arose from the basically incorrect concepts of classical phvsics. This quantum conception of nature has emerged directly and naturally out of the idea of the quantum world that generally prevails today in the minds of practicing quantum physicists: it rests on the idea of Heisenberg and Dirac that under particular kinds of conditions, nature makes a choice. It is based on an examination of the nature of those conditions. The condition under which nature acts was construed as an expression of a criterion of natural value.

It is possible that this criterion of value in natural process applies only at the level of measuring devices. However, it is at least conceivable that the same criterion applies also on other scales, and could be detected as a biasing of quartum choices in favor of the creation of sustainable forms on all levels. Such a biasing should be detectable under laboratory conditions, and may eventually become necessary to introduce into the domains of biology and cosmology, since the ubiquitous existence of sustainable form on all scales may otherwise be impossible to explain in a natural way.

Another possible ramification pertains to the interface between the brain sciences and psychology. It is evident that mental processes are connected in some way to brain processes. However, the nature of the connection is unclear. Indeed, when viewed from the perspective of classical physics such a connection appears totally incomprehensible. For classical physics is fundamentally reductionistic: each macroscopic system is conceived to be nothing more than a simple collection of its microscopic parts, each of which is supposed to react in a completely mechanical way to the instantaneous force that acts upon it. On the other hand, each human experience evidently corresponds to an 'entire enduring complex macroscopic form' in a human brain. According to the concepts of classical physics, no such physical form can exist as a fundamentally unified entity: no such form can exist except as a simple collection of its fundamentally independent microscopic parts. The fundamentally unified complex conscious thought 
has therefore, within the classical conception of nature, nothing of like kind to which it can correspond. Mereover, if some non-physical process of 'perceiving certain features of the brain as a complex whole' is added to the classical picture of nature, in order to account for the occurrence and character of human experience, then this process, if it is not io contradict the laws of classical physics, can have no back-reaction or influence upen the course of physical everits, which is already completely determined, in terms of the motions of the mieroscopic realities, by the deterministic laws of motion.

The quantum mechanical conception of nature is altogether different. In this conception each actual thing is funda nentally the actualization of an entire enduring complex macroscopic form. Those aspects of nature that are described in terms of the simple microscopic parts govern only the tendencies for the actualization of such enduring complex forms. The occurrence of such complex forms is, thersfore, neither incidentail nor external to the basic dynamical process. On the contrary, the actualization of such forms is the entire object of the dynamics, and it is these forms themselves, not the subordinate microscopic parts, that determine what actually happens.

Within the quantum mechanical conception of nature human experiences are, as regards their intrinsic structural forms, similar in kind to the actualities that evidently play the dominant role in high-level brain dynamics. An analysis ${ }^{12}$ of the basic features of higl-level brain functioning, and of conscious mental process, reveals that one can in fact postulate an isomorphism between the intrinsic structure of conscious mental events and the intrinsic structure of a certain class of brain events, conceived of as quantum events. Conscious mental events thereby becorne naturally correlated with events in human brains, as they are described in the language of quantum theory. The occurrence or nonoccurrence of such brain events is, however, not predetermined by the known laws of physics: such decisions are matters of choice.

\section{Summary}

If an important task of science is to provide man with the empirical foundation of a philosophically satisfactory comprehension of the universe, and his place within it, then classical physics is profoundly deficient in two important ways. The first concerns choice and meaning. If a 'choice' is defined to be a 
fixing of an aspect of the universe that is not fixed by the known laws of nature, then at the stage of classical physics all choice is confined to 'the beginning of time': all choice is compressed into some supendous initial act, which arises out of nothing at all, or at least out of nothing representable within the physical theory. The universe is consequently rendered 'meaningless' from the perspective of man, because each human being is reduced to a mecharical automaton whose every action was pre-ordained prior to his own existence.

The advance to quantum theory appears at first to rffer no basis for any significant improvement: choice is now distributed over time, and is confined to particular kinds of physical contexts, but is asserted to be controlled exclusively by 'pure chance'. Thus we are presented with the two horns of the dilemma, 'determinism' or 'chance': neither option appears to offer any possibility for a meaningful universe, or a meaningful role for man within it.

Closer study, however, reveals quite the opposite. An examination of the conditions under which quantum choices are made, according to the "orthodox" ideas of Heisenberg and Dirac, shows that, even though these choices are not fixed by the quantum laws, nonetheless, each such choice is intrinsically meaningful: each quantum choice injects meaning, in the form of enduring structure, into the physical universe.

The second profound deficiency of classical physics is its essentially reductionistic character. According to the concepts of classical physics each thing is essentially nothing more th an a sum of simple parts. But this limitation excludes the possibility of the existence, within the physical universe itself, of a faithful representation of a comprehension of anything; of a representation within the physical universe of anything that mirrors the essential attribute of a conscious thought, namely its existence as a fundamentally complex whole. The fundamental characteristic of a comprehension, or a thought, is precisely that it is more than the sum of its component parts: it cannot be analyzed into nothing more than the sum of its components without eliminating its very essence. Thus within tho physical universe, as classically conceived, there is no possibility of representing a comprehension of anything: one is forced to look outside the classically conceived physical universe to locate human thoughts. On the other hand, it is the essence of Heisenberg/Dirac quantum events that they choose, and actualize within the physical universe itself, as yuantum mechanically con- 
ceived, complex meaningful wholes. Science thus provides man with at least the rudiments of a cohesive vie' $N$ of nature in which his own thoughts and actions are integral parts of a universe that generates meaningful options via the laws of nature, but is not rigidly controlled by these laws.

\section{References}

1. Niels Bohr, Atomic theory and the description of nature, (Cambridge Univ. Press, Cambridge 1934) p.1.

2. Ibid. p.1.

3. Ibid. p.18.

4. Niels Bohr, Atomic physics and human knowledge, (Wiley, New York, 1958) p.90.

5. Niels Bohr, Essays 1958/1962 on atomic physics and human knowledge (Wiley, New york, 1963) p.60.

6. Henry P. Stapp, The Copenhagen Interpretation, Amer. J. of Phys. 40, 1098 (1972).

7. Hugh Everett III, Rev. Mod. Phys. 29, 463 (1957).

8. David Bohm, Phys. Rev. 8.5, 166, 180 (1952).

9. John Bell, On the Einstein-Podolsky-Rusen Paradox, Physics 1, 195 (1964).

10. Henry P. Stapp, Significance or an Experiment of the Greenberger-HorneZeilenger Kind, University of Califonria Lawrence Berkeley Laboratory Report LBL-29377 Nov. 1991.

11. Werner Heisenberg, Physics and Philosophy (Harper and Row, 1958) ch.III.

12. Henry P. Stapp, A quantum theory of the mind-brain interface, Lawrence Berkeley Laboratory Report LBL-28574 Expanded (1990). 

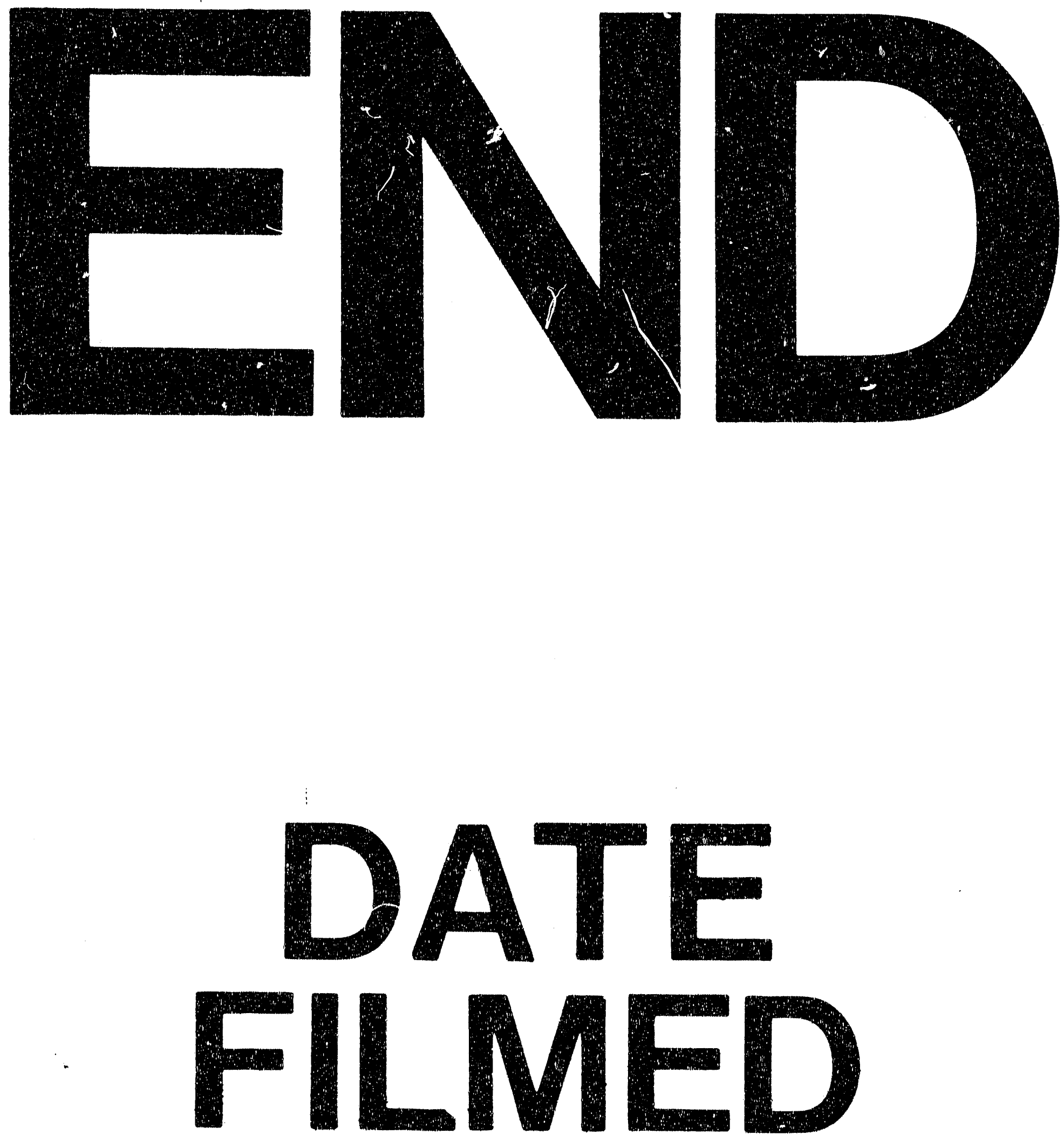

1

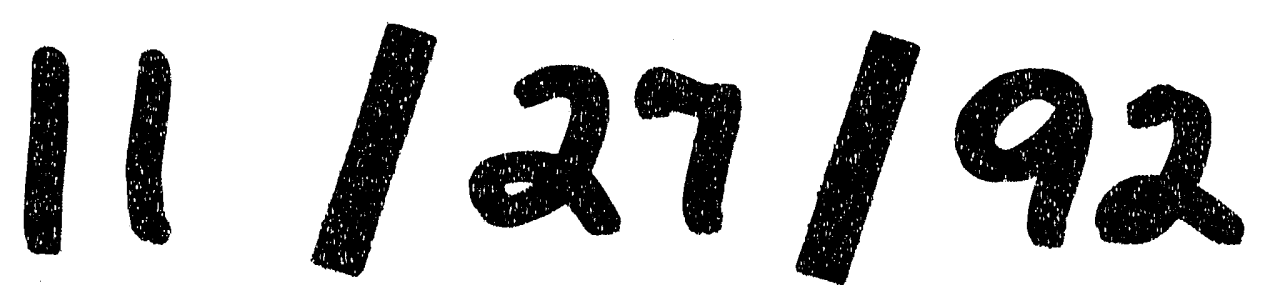


